\title{
Three-Dimensional Point Defect Imaging by Large-angle Illumination STEM
}

Ryo Ishikawa $^{1}$, Stephen J. Pennycook ${ }^{2}$, Andrew R. Lupini ${ }^{3}$, Scott D. Findlay ${ }^{4}$, Naoya Shibata $^{1,5}$ and Yuichi Ikuhara ${ }^{1,5}$

1. Institute of Engineering Innovation, University of Tokyo, Bunkyo, Tokyo 113-8656, Japan

2. Department of Materials Science \& Engineering, National University of Singapore, 177576, Singapore

3. Materials Science and Technology Division, Oak Ridge National Laboratory, TN, 377831, USA

4. School of Physics and Astronomy, Monash University, Victoria 3800, Australia

${ }^{5 .}$ Nanostructures Research Laboratory, Japan Fine Ceramics Center, Nagoya, Aichi 456-8587, Japan

The success of aberration correctors has led to a remarkable improvement in the spatial resolution in scanning transmission electron microscopy (STEM) to better than half an angstrom [1], which is sufficient for determining atomic structure in most materials. However, this high resolution applies only in the lateral two dimensions. The resolution (probe elongation) along the optical axis typically remains several nanometers, hindering the development of three-dimensional atomic-scale imaging in electron microscopy. There are several approaches towards achieving high-resolution 3D imaging and spectroscopy, including electron tomography, hollow-cone illumination STEM and confocal STEM. Though electron tomography has achieved excellent 3D atom-by-atom imaging in some cases [3], it is not well-suited to typical plate-shape thin specimens. Since the successful development of higher-order aberration correctors [2], it has become possible to increase the illumination angle ( $\alpha$ ) up to $70 \mathrm{mrad}$. Therefore, optical depth sectioning with large-angle illumination (LAI) STEM [3] may allow us to solve three-dimensional materials problems. However, two technical difficulties must be overcome to perform atomic-scale optical depth sectioning via LAI-STEM [4]. One difficulty is electron dose. For larger-angle illumination, the electron probe is very finely focused in all three dimensions and we may need to perform observations under relatively low electron dose (shot noise) to avoid excessive specimen damage. The other difficulty is chromatic aberration, where the electron probe is elongated along z-direction by the energy spread of the electrons. In this study, we explore single dopant visibility using LAI-STEM imaging under realistic dose and energy spread conditions through full dynamical image simulations. We also show application of LAI-STEM imaging to thickness measurement and surface imaging [5].

Figure 1 shows simulated ADF-STEM images of Ce-doped $w$-AlN, for the conditions of $300 \mathrm{kV}$ electrons, $\alpha=60 \mathrm{mrad}, C_{c}=1 \mathrm{~mm}$, and $\Delta E=0.3 \mathrm{eV}$. No significant difference is visible between the images with and without chromatic aberration. Figure 2 shows, for a sample $7.4 \mathrm{~nm}$ thick, a cross-sectional-defocusview along the line $\mathrm{X}-\mathrm{X}^{\prime}$ for electron doses of $25 \times 2^{m} \mathrm{e}^{-} /$pix $(m=0,1, \ldots, 7)$. Though the chromatic aberration gives a slight elongation along the $\mathrm{z}$-direction, the bright Z-contrast is well localized within \pm 2 unit cells along z-direction, comparable with the length of point spread function. Therefore, we may efficiently remove the channeling effect by using large-angle illumination. Z-contrast reduction by chromatic aberration is relatively small, enabling focal-series imaging at the low-dose condition of $200 \mathrm{e}^{-}$ /pix. However, for multiple dopants (Fig. 2(c) and (d)), the bright Z-contrast is delocalized along the zdirection, making it difficult not only to determine the dopant depths but even to reliably estimate the number of dopants. Therefore, it will be necessary to develop a new chromatic aberration corrector or better electron source to achieve true 3D atomic-scale imaging. [6] 


\section{References}

[1] H. Sawada et al., Microscopy 64 (2015) 213.

[2] H. Sawada et al., J. Electron Microscopy 58 (2009) 341.

[3] Y. Yang et al., Nature 542 (2017) 75.

[4] R. Ishikawa et al., Ultramicroscopy 151 (2015) 122.

[5] R. Ishikawa et al., Appl. Phys. Lett. 109 (2016) 163102.

[6] A part of this work was supported by the Research \& Development Initiative for Scientific Innovation of New Generation Batteries II (RISING II). A.R.L. was supported by the Office of Basic Energy Sciences, Materials Sciences and Engineering Division, U.S. Department of Energy.

(a)

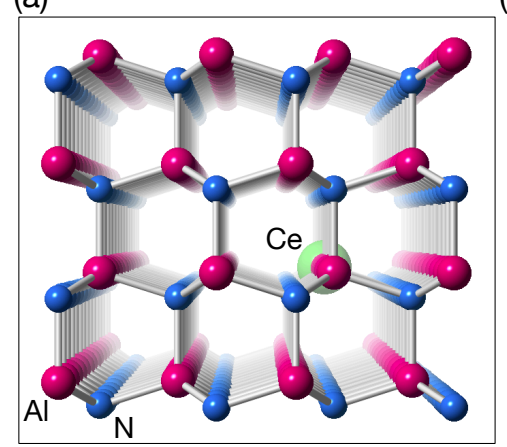

(b) w/o chromatic

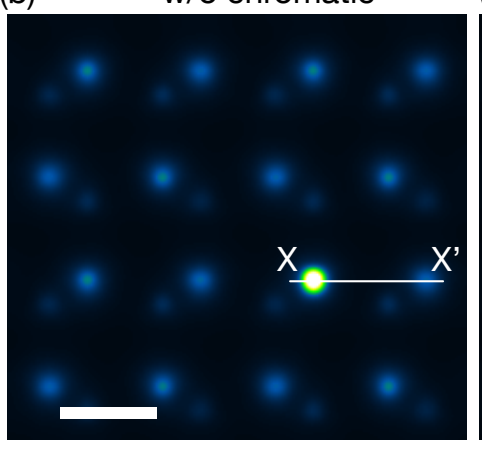

(c) chromatic

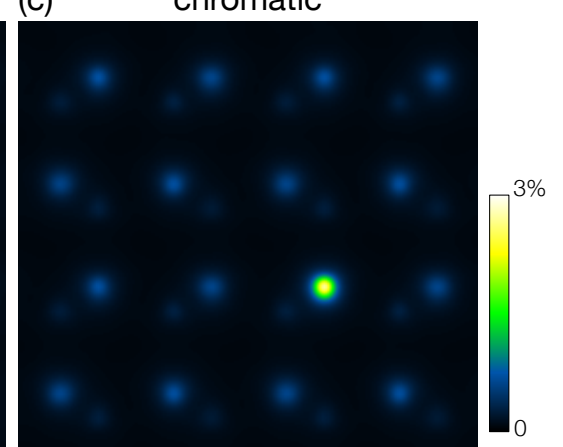

Figure 1. (a) The structure model of Ce-doped $w$-AlN in a-axis projection, for a specimen thickness of $7.4 \mathrm{~nm}$ and a single Ce dopant located $2.1 \mathrm{~nm}$ down from the entrance surface. The simulated ADF-STEM image $(300 \mathrm{kV}, 60 \mathrm{mrad})$ at $\Delta f=2.1 \mathrm{~nm}(\mathrm{~b})$ without and (c) with chromatic aberration $\left(C_{c}=1 \mathrm{~mm}, \Delta E=\right.$ $0.3 \mathrm{eV}$ ). The scale bar in (b) is $3 \AA$.

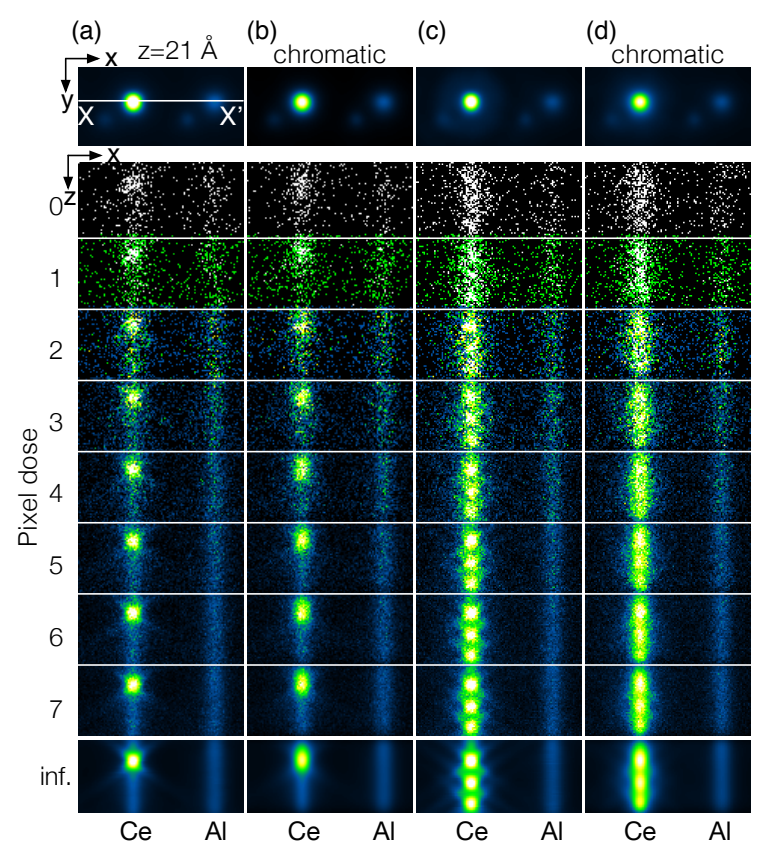

Figure 2. Cross-sectional-defocus-view along the line $X-X$ ' in Fig. 1(b). A single Ce dopant is located at $\mathrm{z}=2.1 \mathrm{~nm}$ in (a), (b) while three Ce dopants are located at $\mathrm{z}=2.1,4.3$ and $6.5 \mathrm{~nm}$ in (c), (d). The different doses are denoted $m=0,1, \ldots, 7$ corresponding to electron doses of $25 \times 2^{m} \mathrm{e}^{-} /$pix, with the bottom row assuming infinite electron dose. Chromatic aberration is included in (b) and (d). 